\section{Medizin allein reicht nicht mehr}

W arum sollte ein Patient ausgerechnet in Ihre Praxis kommen? Wenn Sie die Frage nicht spontan beantworten können, dann sei Ihnen die Lektüre des hier vorgestellten Buches empfohlen.

Die Autorinnen, dem Rezensenten bekannt durch viele sehr gut besuchte Wirtschaftsseminare für niedergelassene Ärzte, sind Diplom-Betriebswirtinnen mit langjähriger Erfahrung in Consulting, Marketing und Praxismanagement. Sie haben in der zweiten Auflage ihres Werkes ihr Konzept für eine erfolgreiche Praxisführung aktualisiert. Dabei zieht sich wie ein roter Faden die Feststellung durch das Buch, dass es heute nicht mehr genügt, nur ein guter Arzt zu sein, sondern dass man eine ganze Menge betriebswirtschaftliche, marketingstrategische und kommuni- kationstechnische Aspekte beachten muss, um seine Praxis erfolgreich zu führen. Dies steht in starkem Widerspruch zu der früher üblichen Auffassung, wonach betriebwirtschaftliches Denken im Medizinstudium nichts zu suchen hat.

Das Buch gliedert sich in die folgenden Abschnitte:

— aktuelle Praxisanalyse,

- Entwicklung einer Marketingstrategie,

- Patienten- und Mitarbeiterorientierung,

- Werbestrategien,

- patientenorientierte Kommunikation,

- patientenorientierte Praxisführung,

- praktische Umsetzung.

Das Buch ist flüssig und unterhaltsam geschrieben, spart nicht mit vielen praktischen Tipps mit zahlreichen Abstechern in die angewandte Psychologie und ist damit eine wichtige Hilfe für den niedergelassenen Arzt zur modernen und erfolgreichen Praxisführung. Gerade in einer Zeit des Umdenkens zur servicebetonten Arztpraxis ist dieses Buch eine wichtige Hilfe für den Praxisinhaber.

Priv.-Doz. Dr. Dr. Thomas A. Angerpointner, München

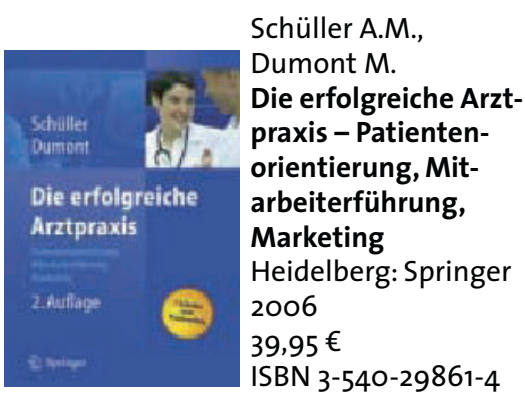

\section{Allergenkarenz leicht gemacht}

S elbst bei bekannter Nahrungsmittelallergie ist es für den Betroffenen nicht einfach, auslösende Stoffe zu meiden. Die neue Lebensmittel-Kennzeichnungsrichtlinie der EU, die in Deutschland im November 2005 in Kraft getreten ist, wird hier zwar eine gewisse Erleichterung bringen, aber die Patienten bedürfen nach wie vor guten Rats, wie sie trotz der Einschränkungen bei der Lebensmittelauswahl eine ausgewogene Ernährung erreichen können.

Nur vier Jahre nach der zweiten Auflage des Buches „Diät bei Nahrungsmittelallergien und -intoleranzen" hat die große Nachfrage jetzt zu einer dritten Auflage geführt. Die Autoren und Herausgeber, allesamt anerkannte Ernährungswissenschaftler oder Allergologen, haben das Gemeinschaftswerk nach neuester Literatur aktualisiert und ergänzt.

Vom Allgemeinen zum Speziellen führt das Buch zunächst in die Problematik der Nahrungsmittelallergie ein. Weiter geht es über das Kapitel Diagnostik hin zu allgemeinen Ernährungs- empfehlungen bei allergischen Erkrankungen. Die nächsten Kapitel geben konkrete Diätempfehlungen bei speziellen Allergien und Unverträglichkeiten gegenüber Kuhmilch, Soja, Hühnereiweiß, Gluten und Weizen, Pollen und Naturlatex, Nickel, Laktose, Fruktose und Sorbit sowie bei Unverträglichkeit gegenüber biogenen Aminen und bei pseudoallergischen Reaktionen. Dazwischen liegen Empfehlungen zum altersabhängigen Kostaufbau bei allergiegefährdeten Kindern und ein Kapitel über die hypoallergene Babynahrung und Atopieprävention. Der Bogen schließt sich mit einem übergreifenden Kapitel zur Ernährungsberatung bei Nahrungsmittelunverträglichkeiten, das auch einen Fragebogen zur Ermittlung des Ernährungsverhaltens von Kindern enthält.

Das Buch besticht durch seine Übersichtlichkeit. Die Kapitel werden jeweils mit einer knappen Quintessenz eröffnet und sind mit insgesamt $35 \mathrm{sehr}$ informativen Tabellen, die geeignete und ungeeignete Nahrungsmittel auflis- ten, gespickt. Genaue Literaturangaben am Ende der Kapitel, wie bei wissenschaftlichen Publikationen üblich, erzeugen Transparenz für diejenigen, die gerne weiter in die Tiefe gehen wollen und diese Angaben bei vielen Patientenratgebern bisher vergeblich suchten. Ein kleines Glossar, in dem Laien die wichtigsten Fachbegriffe erklärt werden, rundet das Werk ab.

Das Werk fußt auf den wissenschaftlichen Erkenntnissen der modernen Allergologie und Ernährungswissenschaft und sieht seine vornehmste Aufgabe in der praktischen Beratung von Betroffenen. Aber auch für Fachund Hausärzte sowie für Ernährungs berater kann es durchaus als Nachschlagewerk dienen.

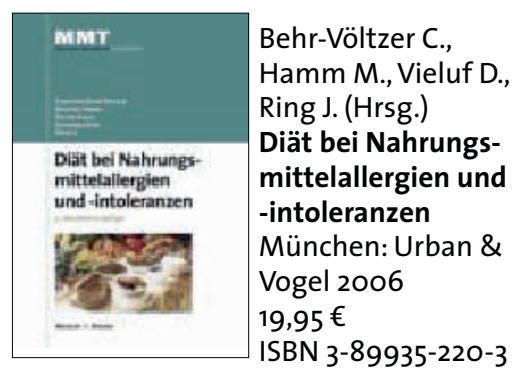

